\title{
IBPSO-Based MUSIC Algorithm for Broken Rotor Bars Fault Detection of Induction Motors
}

\author{
Pan-Pan Wang ${ }^{1 *}$, Xiao-Xiao Chen ${ }^{1}$, Yong Zhang ${ }^{2}$, Yong-Jun $\mathrm{Hu}^{1}$ and Chang-Xin Miao ${ }^{1}$
}

\begin{abstract}
In spectrum analysis of induction motor current, the characteristic components of broken rotor bars (BRB) fault are often submerged by the fundamental component. Although many detection methods have been proposed for this problem, the frequency resolution and accuracy are not high enough so that the reliability of BRB fault detection is affected. Thus, a new multiple signal classification (MUSIC) algorithm based on particle swarm intelligence search is developed. Since spectrum peak search in MUSIC is a multimodal optimization problem, an improved bare-bones particle swarm optimization algorithm (IBPSO) is proposed first. In the IBPSO, a modified strategy of subpopulation determination is introduced into BPSO for realizing multimodal search. And then, the new MUSIC algorithm, called IBPSO-based MUSIC, is proposed by replacing the fixed-step traversal search with IBPSO. Meanwhile, a simulation signal is used to test the effectiveness of the proposed algorithm. The simulation results show that its frequency precision reaches $10^{-5}$, and the computational cost is only comparable to that of traditional MUSIC with 0.1 search step. Finally, the IBPSO-based MUSIC is applied in BRB fault detection of an induction motor, and the effectiveness and superiority are proved again. The proposed research provides a modified MUSIC algorithm which has sufficient frequency precision to detect BRB fault in induction motors.
\end{abstract}

Keywords: MUSIC, Multimodal, Bare-bones particle swarm optimization, Induction motors, Broken rotor bars, Fault detection

\section{Introduction}

Induction motors are essential components and play an irreplaceable role in industrial production process [1]. Although this motor is simple-structure, low-cost, reliable and robust, it is also prone to failure due to reasons, such as harsh working environment, internal incipient defects [2]. Broken rotor bars (BRB) or end ring cracking is one of the most common faults of induction motor, which accounts for about $10 \%$ of all failures [3]. This percentage is inconspicuous, but BRB fault will not only reduce the efficiency, but also cause other faults, such as bearing fault, air-gap eccentricity [4]. In severe cases, broken pieces of the rotor bar may even damage the stator windings during operation [5]. Therefore, rotor fault

\footnotetext{
*Correspondence: wpp2011@126.com

${ }^{1}$ School of Electrical and Power Engineering, China University of Mining \& Technology, Xuzhou 221116, China

Full list of author information is available at the end of the article
}

detection of induction motor in early stage is essential and significant.

When broken bars occur, new sideband components at $f_{\text {brk }}=(1 \pm 2 k s) f_{1} \mathrm{~Hz}$ will appear in the stator current spectrum, where $k=1,2,3, \ldots, f_{1}$ is the supply frequency and $s$ is the motor slip [6]. So the sideband components can be regarded as fault characteristics, and their frequency detection is key to identify the BRB fault. However, the strongest fault-related components at $(1 \pm 2 s) f_{1} \mathrm{~Hz}$ are quite close to fundamental component due to small slip value and their amplitudes are relatively small, which make them easy to be submerged by the leakage of fundamental component, reducing the reliability of BRB fault detection. In addition, their signal property differs significantly under various motor-working conditions. When motor runs in steady state, the values of $f_{\mathrm{brk}}$ are almost constant, and when motor is running in transient state, such as start up or load change, the values of $f_{\text {brk }}$ are variable, that is to say, the fault-related components are non-stationary signals. For these two different properties 
of stator current, scholars have adopted many different signal processing methods.

In transient state, promising results have been obtained through approaches based on short-time Fourier transform [7], continuous or discrete wavelet transform [7, 8], fractional Fourier transform [9], complex empirical mode decomposition [10] and Synchrosqueezing transform [11]. Yet, the frequency resolution and accuracy of these methods are low, which restrict their applications.

For steady state of induction motor, spectrum analysis based on discrete Fourier transform (DFT) is the most popular method for BRB fault detection. However, in this case, the influence of spectrum leakage of fundamental component is more serious due to smaller slip value. To solve this problem, many distinctive methods have been developed, such as instantaneous power [12], particle swarm optimization [13] and Hilbert transform [14]. The main idea of these methods is to filter the fundamental component or convert it into a DC component from current signal. Then DFT is implemented to highlight the fault characteristics. Although these methods based on DFT analysis can eliminate the influence of fundamental component, their performances are restricted by DFT own shortcomings. For example, the frequency resolution is limited by measurement time, namely, high frequency resolution needs to be ensured with a long enough measurement time. If the signal measurement period is too long, the probability of load fluctuations, noise and other interference factors will be increased, which will affect the accuracy of fault detection [15].

As one of the modern spectrum estimation methods, multiple signal classification (MUSIC) algorithm has a capability of original signal extrapolation and high frequency resolution. Therefore, MUSIC has been introduced into the BRB fault detection of induction motor. Garcia-Perez et al. [16] applied MUSIC to multiple faults detection of induction motor. On the basis of MUSIC, Fang et al. [17] moved the maximum eigenvector of fundamental component into noise subspace to constitute a new noise space. The results indicate that this method not only eliminates the influence of fundamental component, but also improves the frequency resolution. ZMUSIC method based on the frequency spectrum zooming technique and MUSIC was presented by Kia et al. [18], to further improve the frequency resolution and computational efficiency. A hybrid scheme based on MUSIC and empirical mode decomposition was proposed by Camarena-Martinez et al. [19], to detect multiple faults of induction motor. Romero-Troncoso et al. [20] detected rotor unbalance fault using a hybrid MUSIC method combined with complete-ensemble-EMD. Another method that combined optimization algorithm with MUSIC was presented by $\mathrm{Xu}$ et al. [21]. It solved the problem that MUSIC couldn't compute the amplitudes and phases of components. However, when the MUSIC is used in BRB fault detection, its efficiency of spectrum peak search is low due to fixed-step and traversal search.

Particle swarm optimization (PSO) is an emerging global optimization technology proposed by Kennedy et al. [22], in 1995. Because of the advantages of simple concept, easy realization, effective solution to complex problems, PSO has been widely applied in practical engineering problems, such as structural optimization [23], design optimization, PID controller tuning, stretch force trajectory optimization and parameter optimization [24]. However, the standard PSO and most improved algorithms are designed to deal with the problem which includes only one global optimal solution in search space. They are not suitable for searching multiple extremes, such as searching spectrum peaks in MUSIC. To optimize multimodal problem, scholars have introduced niche technology into evolutionary algorithm, and accordingly presented many strategies $[25,26]$, such as pre-selection technique, crowding strategy, fitness sharing, species conservation, but several issues still remain. For instance, low convergence speed and precision, trapped in local optimum and error fluctuation may compromise the optimization results.

This paper tries to improve PSO and take the advantages of PSO and MUSIC for BRB detection in induction motor. Organization of this paper is as follows. In Section 2, the principles of MUSIC are introduced firstly. In Section 3, an improved bare-bones PSO (IBPSO) is proposed for multimodal optimization problem. In Section 4, a new MUSIC method based on IBPSO is proposed and its simulation analysis is also conducted. In Section 5, the IBPSO-based MUSIC is applied in BRB fault detection and its performance is compared with DFT and traditional MUSIC.

\section{Multiple Signal Classification}

As a kind of frequency estimation technique based on matrix eigenvalue decomposition, multiple signal classification (MUSIC) was proposed by Schmid [27] in the 1980s. Its main idea is described below. Firstly, through eigenvalue decomposition, the information space of observed signal is divided into two orthogonal subspaces, namely, signal subspace and noise subspace. Then the frequency components in observed signal can be estimated by constructing spectrum according to subspaces' orthogonality.

Assuming that observed signal $y(n)$ consists of $P$ complex sinusoidal signals and white noise, it is expressed as 


$$
y(n)=\sum_{q=1}^{P} A_{q} \exp \left[\mathrm{j}\left(2 \pi_{q} n+\phi_{q}\right)\right]+h(n) .
$$

Where $n=1,2, \ldots, N, N$ is the number of sampling points, $A_{q}, f_{q}, \phi_{q}$ are amplitude, frequency and initial phase of the $q$ th sinusoidal signal respectively, $h(n)$ is a complex white noise with a zero mean and a variance $\tau^{2}$.

For the observed signal of Eq. (1), a $M \times N$ matrix is constructed as follows $(M>>P)$

$$
\boldsymbol{Y}(n)=[y(n), y(n+1), \ldots, y(n+M-1)] .
$$

Its autocorrelation matrix is

$$
\boldsymbol{R}_{y y}=\mathrm{E}\left\{\boldsymbol{Y}(n) \boldsymbol{Y}^{\mathrm{T}}(n)\right\},
$$

where superscript $\mathrm{T}$ represents the conjugate transpose of matrix, $\mathrm{E}$ is digital expectation.

Then, all eigenvalues decomposed from $\boldsymbol{R}_{y y}$ are sorted in the descending order. The subspace spanned by eigenvectors corresponding to the $P$ largest eigenvalues is the signal subspace $S$. The subspace spanned by the rest of eigenvectors is the noise subspace $\boldsymbol{G}$.

Since subspaces $\boldsymbol{S}$ and $\boldsymbol{G}$ are orthogonal, signal frequency vectors and noise subspace are also orthogonal, that is

$$
\boldsymbol{D}\left(f_{q}\right) \perp \boldsymbol{G},
$$

where $\boldsymbol{D}\left(f_{q}\right)=\left[1, \exp \left(\mathrm{j} 2 \pi f_{q}\right), \exp \left(\mathrm{j} 4 \pi f_{q}\right), \ldots, \exp \left(\mathrm{j} 2 \pi M f_{q}\right)\right]$.

For frequency $f_{q}$ included in signal $y(n)$, since $\boldsymbol{D}\left(f_{q}\right)^{\mathrm{T}} \cdot \boldsymbol{G}=0$, then $P M\left(f_{q}\right)=1 /\left|\boldsymbol{D}\left(f_{q}\right)^{\mathrm{T}} \cdot \boldsymbol{G}\right|^{2}$ will take a peak at $f_{q}$. Therefore, signal frequency estimation can be obtained by searching peaks of $P M\left(f_{q}\right)$ with step $\Delta f$.

\section{Improved Bare-Bones Particle Swarm Optimization}

\subsection{Particle Swarm Optimization}

Particle swarm optimization (PSO) is a global optimization technique. It was developed by Kennedy and Eberhart, according to the simulation of social behavior of natural biological groups. In PSO algorithm, there are many particles flying around in a multi-dimension search space. Each particle represents a candidate solution of the problem and adjusts its position based on two optima. One is its personal best position $\boldsymbol{p}_{i}$, namely, its own flying experiences, while the other is the global best position $\boldsymbol{p}_{\mathrm{g}}$, namely, flying experiences of companions. Meanwhile, each particle also corresponds to a fitness value determined by optimization function. Since PSO is a iterative optimization algorithm, in each generation, the velocity $\boldsymbol{v}_{i}=\left[v_{i 1}, v_{i 2}, \ldots, v_{i \beta}\right]$ and position $\boldsymbol{x}_{i}=\left[x_{i 1}, x_{i 2}, \ldots, x_{i \beta}\right]$ of each particle are updated by the following formulas [22],

$$
\begin{aligned}
v_{i j}(m+1)= & w v_{i j}(m)+c_{1} r_{1}\left(p_{i j}(m)-x_{i j}(m)\right) \\
& +c_{2} r_{2}\left(p_{g j}(m)-x_{i j}(m)\right), \\
x_{i j}(m+1)= & x_{i j}(m)+v_{i j}(m+1),
\end{aligned}
$$

where $w$ is an inertia weight to control exploration in the search space, $c_{1}$ and $c_{2}$ are two learning factors, $r_{1}$ and $r_{2}$ are two random numbers within $(0,1),-v_{\max } \leq v_{i j} \leq v_{\max }$, and $v_{\max }$ is the maximum velocity set by the user, $i=1,2$, $\ldots, \alpha, \alpha$ is the population size of particle swarm, $j=1,2$, $\ldots, \beta, \beta$ is the dimension of search space.

\subsection{Bare-Bones Particle Swarm Optimization}

Clerc and Kennedy [28] analyzed the trajectory of particles, and proved that each particle $\boldsymbol{x}_{i}$ converges to $\boldsymbol{B}_{i}$ which is a weighted average of its personal best and global best in the standard PSO algorithm, that is

$$
B_{i j}=\frac{c_{1 j} p_{i j}+c_{2 j} p_{\mathrm{g} j}}{c_{1 j}+c_{2 j}} .
$$

where $c_{1 j}$ and $c_{2 j}$ are two random numbers within $(0,1)$. When iteration number tends to infinity, all the particles will converge to the same point.

Based on above, Kennedy [29] proposed the bare-bones particle swarm optimization (BPSO) in 2003. In BPSO, a Gaussian distribution based on the $\boldsymbol{p}_{i}$ and $\boldsymbol{p}_{\mathrm{g}}$ is used to update particle's position. The update formula is

$$
x_{i j}(m+1)=\chi\left(\mu_{i j}(m), \eta_{i j}^{2}(m)\right),
$$

where $\mu_{i j}(m)=\left(p_{i j}(m)+p_{\mathrm{g} j}(m)\right) / 2$ is the mean of Gaussian distribution, and $\eta_{i j}^{2}(m)=\left|p_{i j}(m)-p_{\mathrm{gj}}(m)\right|$ is the standard deviation.

Compared with the standard PSO, BPSO needs less control parameters, and that is more suitable for practical application.

\subsection{Improved BPSO}

In MUSIC, the spectrum peak search is a multimodal optimization problem that needs to search for all global optimum solutions and as many local optimum solutions as possible. For this problem, a novel strategy of subpopulation determination based on the excellent work of Refs. [25, 26] is proposed in this section, and then is introduced into BPSO to realize multimodal search. Compared with Refs. [25, 26], the improved BPSO (IBPSO) has three differences: (1) Personal best 
position is selected as a candidate of seeds to prevent oscillation; (2) A similarity of species $\sigma$ is defined for maintaining the diversity of seeds, where $\sigma$ is a Euclidean distance between any two particles; (3) Each seed particle is the core of subpopulation, and non-seed particles are distributed to the subpopulation of the nearest seed. The pseudo code of improved seed selection strategy is shown as follows:

Input: Spbest-containing all personal best positions sorted in decreasing order fitness.

1) begin

2) $\quad \mathbf{X}_{\mathbf{s}}=\boldsymbol{\Phi}$ (null set);

3) while (not reaching the end of Spbest) do

4) Get best unprocessed $\boldsymbol{p} \in$ Spbest;

5) found $\leftarrow$ FALSE;

6) for all $x^{*} \in \mathbf{X}_{\mathrm{s}}$ do

7) if $\left(\operatorname{distance}\left(\boldsymbol{x}^{*}, \boldsymbol{p}\right) \leq \sigma^{*}\right)$ then

8) found $\leftarrow$ TRUE;

9) break;

10) end (if)

11) end (for)

12) if (not found) then

13) $\quad$ let $\mathbf{X}_{\mathrm{s}} \leftarrow \mathbf{X}_{\mathrm{s}} \cup\{\boldsymbol{p}\}$;

14) end (if)

15) end (while)

16) end

Output: $\mathbf{X}_{\mathbf{s}}$-containing dominating particles identified as subpopulation seeds.

As described above, seed selection strategy is executed in each generation of the evolutionary process. First of all, according to the fitness value, personal best positions of all particles are arranged in descending order to form a set Spbest. Then, the similarity between each individual in Spbest and each existing seed of $\mathbf{X}_{\mathbf{s}}$ (called seed set) is compared. If the Euclidean distance between them is larger than threshold value $\sigma^{*}$, this personal best position will be added to $\mathbf{X}_{\mathbf{s}}$.

The basic steps of IBPSO are as follows:

Step 1: Initialize particles' positions, personal best positions and seed set $\mathbf{X}_{\mathbf{s}}$. Set algorithm parameters, including population size $\alpha$, maximum generation $m_{\max }$ and species similarity threshold $\sigma^{*}$

Step 2: Calculate the fitness of each particle

Step 3: Update personal best position $\boldsymbol{p}_{i}$

Step 4: According to the fitness, arrange all $\boldsymbol{p}_{i}$ in descending order

Step 5: Update seed set $\mathbf{X}_{\mathbf{s}}$
Step 6: Assign each particle to the nearest seed by calculating the distance between them

Step 7: According to Eq. (8), update particle positions, where $\boldsymbol{p}_{\mathrm{g}}$ is the seed found in Step 5;

Step 8: If the condition of termination is satisfied (i.e., the fitness error is less than the threshold or the iterative number exceeds the maximum), stop iterative procedure and output all the seed positions and fitness values. Otherwise, return to Step 2

\subsection{IBPSO Performance Analysis}

A benchmark function, Rastrigin, is used to verify the effectiveness of IBPSO. And the performance of IBPSO is compared with species conserving genetic algorithm (SCGA) [25] and species-based PSO (SPSO) [26].

In mathematical optimization, Rastrigin function is a typical example of non-linear multimodal function and often used as a performance test problem for optimization algorithms. For two independent variables, it is defined as

$$
\min F\left(x_{1}, x_{2}\right)=\sum_{j=1}^{2}\left[x_{j}^{2}-10 \cos \left(2 \pi x_{j}\right)+10\right],
$$

where $x_{1}, x_{2} \in[-5,5]$. Three-dimensional diagram of Eq. (9) is shown in Figure 1. As the plot shows, Rastrigin function has large search space and large number of local minima. So solving this multimodal optimization is a fairly difficult task.

In this test, the target of three algorithms is to find one global optimum and eight local optima with best fitness. Their parameter configurations are shown in Table 1 . With the same initial population, three algorithms are performed on above benchmark function. The results are shown in Figures 2 and 3.

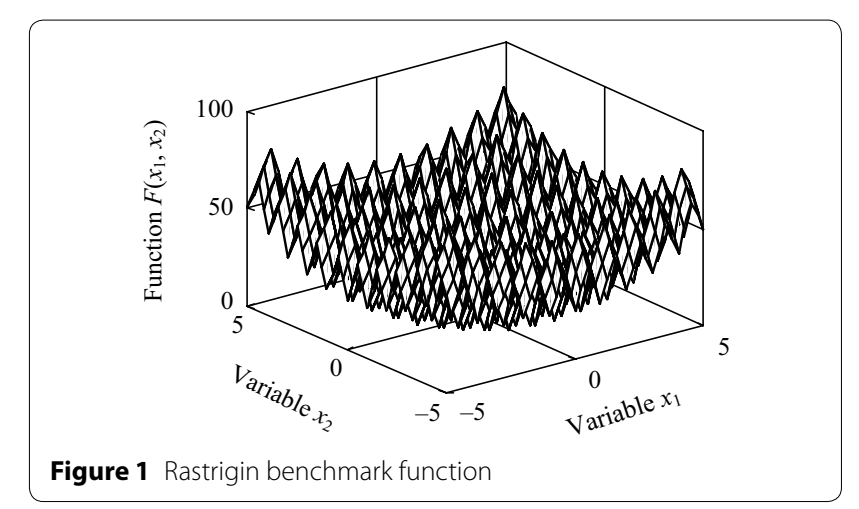


Table 1 Parameter configurations for the selected optimization algorithms

\begin{tabular}{llll}
\hline Parameter & SCGA & SPSO & IBPSO \\
\hline Population size $a$ & 150 & 150 & 150 \\
Maximum generation $m_{\max }$ & 50 & 50 & 50 \\
Species similarity threshold $\sigma^{*}$ & - & - & 0.9 \\
Niche radius $r_{\mathrm{n}}$ & 1.5 & 1.25 & - \\
Crossover probability $\gamma_{\mathrm{c}}$ & 0.6 & - & - \\
Mutation probability $\gamma_{\mathrm{m}}$ & 0.05 & - & - \\
Constriction factor $\lambda$ & - & 0.729844 & - \\
Learning factor $c_{1}, c_{2}$ & - & 2.05 & - \\
\hline
\end{tabular}

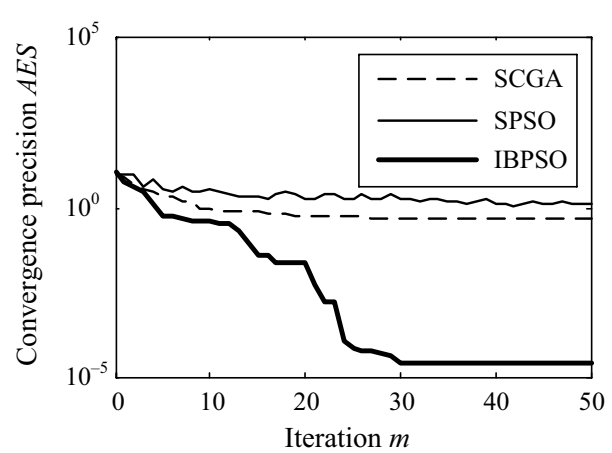

Figure 2 Convergence curves of three algorithms

As shown in Figure 2, the performance of IBPSO is the best, SCGA second, whereas SPSO worst. There is an error fluctuation on the convergence curve of SPSO, because its seeds are selected from the particles in each generation. Conversely, SCGA and IBPSO respectively adopt the species conservation strategy and improved seed selection strategy to effectively avoid this problem. We also can see that IBPSO is better than the other two algorithms both in convergence speed and precision. In Figure 2, the convergence precision $A E S$ is defined as

$$
A E S=\frac{1}{S N} \sum_{i=1}^{S N}\left|F\left(\boldsymbol{k g o}_{\boldsymbol{i}}\right)-F\left(\boldsymbol{s d}_{\boldsymbol{i}}\right)\right|,
$$

where $S N$ is the number of known global optima, $F(\cdot)$ is the fitness function. A pair of $\boldsymbol{k g \boldsymbol { o } _ { \boldsymbol { i } }}$ and $\boldsymbol{s \boldsymbol { d } _ { i }}$ represents that for each real optimum $\boldsymbol{k g o}_{\boldsymbol{i}}$, there is correspondingly a closest species seed $\boldsymbol{s d _ { i }}$ to $\mathbf{k g o}_{\boldsymbol{i}}$.

As shown in Figure 3(a) and (b), for the given nine optimal solutions, SCGA finds eight, and SPSO finds only five. Unfortunately, the SPSO also fail to find the global optimal solution $(0,0)$ with best fitness. The reason is that they adopt fixed niche radius to determine subpopulations, which limits the global search ability of algorithm. On the contrary, IBPSO has an improvement. It can adaptively adjust subpopulations according to the distance between each particle and seed. This behavior makes particles have a larger flight range at the beginning of iteration, which can improve the global search ability. And with the increasing generations, search radius can be reduced automatically to improve the local search ability. Figure 3(c) verifies that IBPSO finds all the optimal solutions with high location accuracy.

In addition, only species similarity threshold $\sigma^{*}$ needs to be set in IBPSO so that the effect of parameters will be reduced. So the IBPSO is more suitable for application in engineering.

\section{IBPSO-Based MUSIC and Simulation Analysis 4.1 IBPSO-Based MUSIC}

In traditional MUSIC algorithm, we need to pre-set the calculation step $\Delta f$ of peak search. And then a traversal search with step $\Delta f$ is performed in the whole frequency domain or within an empirical frequency range. However, this search strategy has two shortages. (1) Since search step $\Delta f$ is fixed, frequency resolution is limited; (2) With decreasing search step, computational time will make a sharp increase, which influences the real-time performance of algorithm. In this section, a new MUISC algorithm combining the traditional MUSIC and IBPSO is proposed, which can fast find multiple spectrum peaks with high precision. To achieve multiple peaks search by IBPSO, an objective function (fitness function) must be established first. According to the principles of MUSIC, fitness function is defined as

$$
\text { Fitness }\left(f_{q}\right)=\left|\boldsymbol{D}\left(f_{q}\right)^{\mathrm{T}} \cdot \boldsymbol{G} \cdot \boldsymbol{G}^{\mathrm{T}} \cdot \boldsymbol{D}\left(f_{q}\right)\right| .
$$

And then the fixed-step traversal search is replaced by IBPSO to improve the performances of MUSIC. The specific steps of IBPSO-based MUSIC are as follows.

Step 1: Measure a set of signal data, and construct its autocorrelation matrix $\boldsymbol{R}_{y y}$

Step 2: Generate the signal subspace $S$ and noise subspace $\boldsymbol{G}$ by handling $\boldsymbol{R}_{y y}$ with eigenvalue decomposition.

Step 3: Encode the $f_{q}$ of Eq. (1) to form population particles, and select the Eq. (11) as the fitness function.

Step 4: Search multiple spectrum peaks in the frequency domain by using IBPSO. 


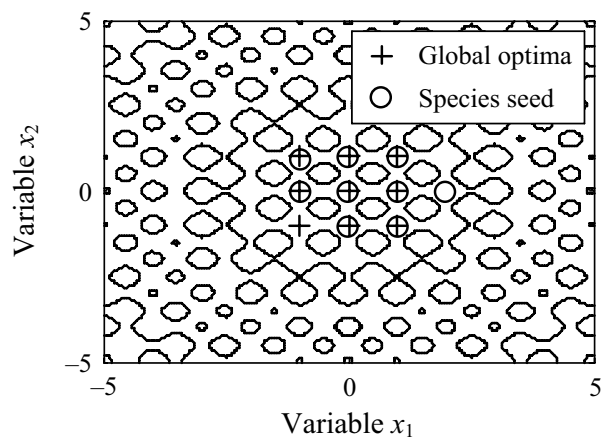

a SCGA

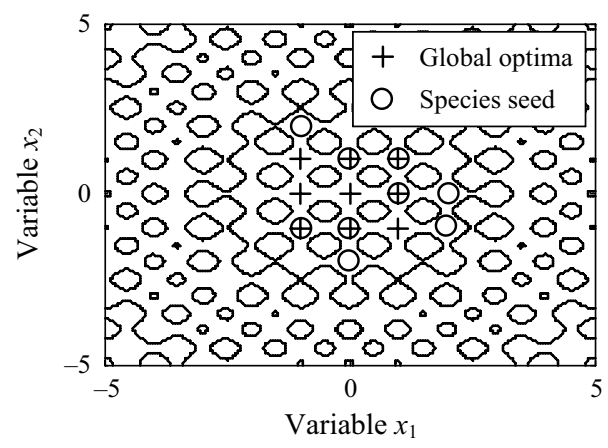

b SPSO

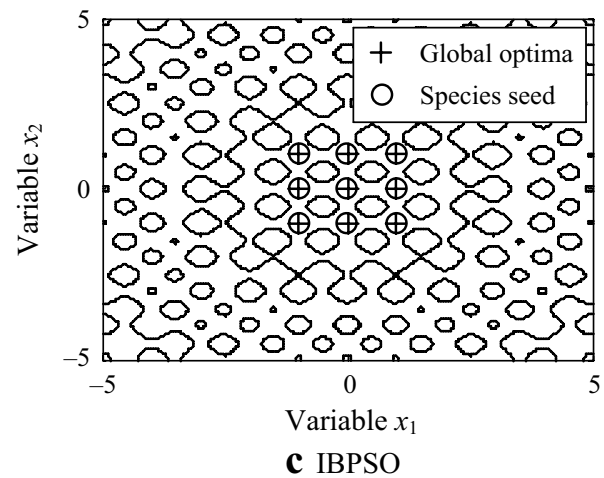

Figure 3 Optimizing results of three algorithms

Table 2 MUSIC estimation results with different search step

\begin{tabular}{|c|c|c|c|c|}
\hline \multirow[t]{2}{*}{ Frequency parameter } & \multirow[t]{2}{*}{ True value } & \multicolumn{3}{|c|}{$\begin{array}{l}\text { Estimation value } \\
\text { with different search step }\end{array}$} \\
\hline & & $\Delta f=0.1$ & $\Delta f=0.01$ & $\Delta f=0.001$ \\
\hline $\begin{array}{l}\text { Fundamental frequency } \\
f_{1}(\mathrm{~Hz})\end{array}$ & 50.0000 & 50.0 & 50.00 & 50.000 \\
\hline $\begin{array}{l}\text { Fault-related frequency } \\
\qquad(1-2 s) f_{1}(\mathrm{~Hz})\end{array}$ & 49.5438 & 49.6 & 49.54 & 49.544 \\
\hline $\begin{array}{l}\text { Fault-related frequency } \\
\qquad(1+2 s) f_{1}(\mathrm{~Hz})\end{array}$ & 50.4562 & 50.5 & 50.46 & 50.456 \\
\hline
\end{tabular}

\subsection{Simulation Analysis}

When the BRB fault occurs in induction motor, sideband components will be generated in the stator current. Among these fault-related components, amplitudes of $f_{\text {br1 }}=(1 \pm 2 s) f_{1}$ are maximum. Thus, to analyze the performance of the IBPSO-based MUSIC in BRB fault detection, the simulated current signal is designed as

$$
\begin{aligned}
i_{\mathrm{br}}(t)= & I_{1} \cos \left(2 \pi f_{1} t+\phi_{1}\right)+I_{\mathrm{bp} 1} \cos \left[2 \pi(1-2 s) f_{1} t+\phi_{\mathrm{bp} 1}\right] \\
& +I_{\mathrm{bn} 1} \cos \left[2 \pi(1+2 s) f_{1} t+\phi_{\mathrm{bp} 1}\right]+h(t) .
\end{aligned}
$$

where $I_{1}, f_{1}, \phi_{1}$ are amplitude, frequency and initial phase of fundamental current, and their values are 10,50 and $\pi / 4$, respectively. Set $I_{\mathrm{bp} 1}=I_{\mathrm{bn} 1}=0.2 \mathrm{~A}$, $(1-2 s) f_{1}=49.5438 \mathrm{~Hz},(1+2 s) f_{1}=50.4562 \mathrm{~Hz}, \phi_{\mathrm{bp} 1}=\pi / 2$ $\mathrm{rad}, \phi_{\mathrm{bn} 1}=\pi \mathrm{rad} . h(t)$ is uniformly distributed random noise signal within $(-0.2,0.2)$. The sampling frequency of simulated current is $250 \mathrm{~Hz}$, and sampling length is 500. Then traditional MUSIC and the proposed algorithm are applied to this signal. In traditional MUSIC, search step $\Delta f$ is set to $0.1 \mathrm{~Hz}, 0.01 \mathrm{~Hz}, 0.001 \mathrm{~Hz}$, respectively, and the simulation results are shown in Table 2 . In the IBPSO-based MUSIC, its related parameters are set as follows: population size is 60 , maximum iterative number is 30 , species similarity threshold $\sigma^{*}=0.3$. Since IBPSO is a stochastic optimization algorithm, the proposed method runs 50 times independently. Its statistical results are shown in Table 3.

Table 2 shows that traditional MUSIC has a very high frequency resolution even with a short-time data window. Therefore, it has superiority in the stator current spectrum analysis, especially in BRB fault detection of induction motor. However, its estimation accuracy of frequency is limited by search step, that is, the highest accuracy will not exceed $\Delta f$. And the increasing search step results in increasing error of the faultrelated frequencies. Fortunately, it is an exception for fundamental frequency, because $50 \mathrm{~Hz}$ is an integer multiple of the search steps like 0.1, 0.01, and 0.001. For the IBPSO-based MUSIC, the estimation accuracy of fault-related frequencies reaches $10^{-5}$, even $10^{-7}$ for fundamental frequency, as Table 3 shown. And the maximum error in 50 times of operation doesn't exceed $10^{-4}$. Therefore, in terms of precision, the proposed algorithm has far exceeded the traditional MUSIC with $\Delta f=0.001 \mathrm{~Hz}$.

Table 4 compares the two algorithms' computational costs. For the MUSIC, the calculation time will increase sharply with the decrease of search step. Namely, the improvement of accuracy is at the expense of computation. For the proposed algorithm, not only the frequency precision reaches $10^{-5}$ (as shown in Table 3), 
Table 3 Estimation results of IBPSO-based MUSIC

\begin{tabular}{|c|c|c|c|c|}
\hline \multirow[t]{2}{*}{ Frequency parameter } & \multirow[t]{2}{*}{ True value } & \multicolumn{3}{|l|}{ Statistical indices } \\
\hline & & $\begin{array}{l}\text { Average estimation } \\
\text { value }\end{array}$ & $\begin{array}{l}\text { Average estimation } \\
\text { error }\end{array}$ & $\begin{array}{l}\text { Maximum } \\
\text { estimation } \\
\text { error }\end{array}$ \\
\hline Fundamental frequency $f_{1}(\mathrm{~Hz})$ & 50.0000 & 50.0000 & $4.7 \times 10^{-7}$ & $4.8 \times 10^{-5}$ \\
\hline Fault-related frequency $(1-2 s) f_{1}(\mathrm{~Hz})$ & 49.5438 & 49.5438 & $1.1 \times 10^{-5}$ & $7.8 \times 10^{-4}$ \\
\hline Fault-related frequency $(1+2 s) f_{1}(\mathrm{~Hz})$ & 50.4562 & 50.4562 & $6.9 \times 10^{-6}$ & $8.4 \times 10^{-4}$ \\
\hline
\end{tabular}

Table 4 Computation cost comparison for the algorithms

\begin{tabular}{|c|c|c|c|c|}
\hline \multirow[t]{2}{*}{ Index } & \multicolumn{3}{|c|}{$\begin{array}{l}\text { MUSIC with different search } \\
\text { step }\end{array}$} & \multirow{2}{*}{$\begin{array}{l}\text { IBPSO-based } \\
\text { MUSIC (average } \\
\text { time in } 50 \text { runs) }\end{array}$} \\
\hline & $\Delta f=0.1$ & $\Delta f=0.01$ & $\Delta f=0.001$ & \\
\hline $\begin{array}{l}\text { Computation time } \\
t(\mathrm{~s})\end{array}$ & 0.101 & 0.814 & 7.732 & 0.339 \\
\hline
\end{tabular}

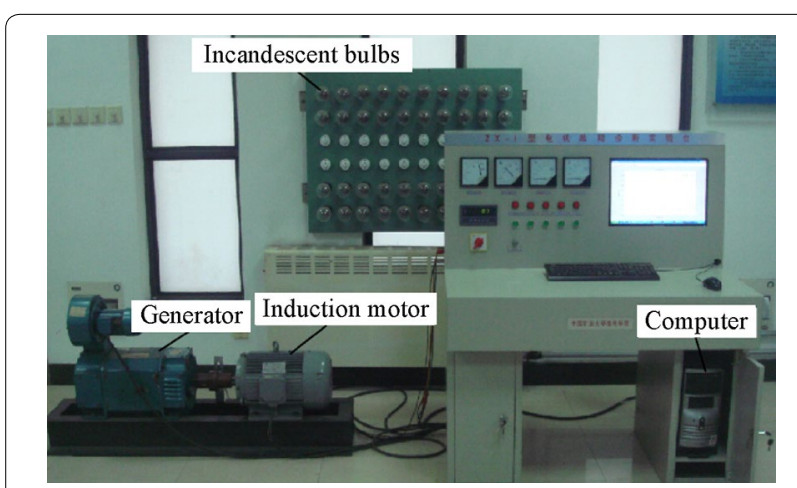

Figure 4 Experimental test bench

and the calculation cost is only $0.339 \mathrm{~s}$ that is equivalent to the traditional MUSIC with $\Delta f=0.1 \mathrm{~Hz}$.

Therefore, IBPSO-based MUSIC obtains a prominent improvement on solution accuracy and search efficiency contrasting with traditional MUSIC.

\section{Application in BRB Fault Detection of Induction Motors}

In this section, IBPSO-based MUSIC is applied in BRB fault detection of an actual induction motor. The general view of the experimental setup is shown in Figure 4. The motor drives an $8 \mathrm{~kW}$ generator to supply 20 sets of incandescent bulbs. The incandescent bulb groups can be switched to adjust the motor load as required. With three Hall current sensors, current signals are acquired into the computer for detection and analysis by the analog signal acquisition card PCI8622.
Table 5 Specifications of the test motor

\begin{tabular}{lllll}
\hline $\begin{array}{l}\text { Rated } \\
\text { power } \boldsymbol{P}_{\mathbf{N}} \\
(\mathrm{kW})\end{array}$ & $\begin{array}{l}\text { Rated } \\
\text { voltage } U_{\mathbf{N}} \\
(\mathrm{V})\end{array}$ & $\begin{array}{l}\text { Rated } \\
\text { current } I_{\mathbf{N}} \\
(\mathrm{A})\end{array}$ & $\begin{array}{l}\text { Rated } \\
\text { speed } \boldsymbol{n}_{\mathbf{N}}(\mathrm{r} /\end{array}$ & $\begin{array}{l}\text { Number } \\
\text { of rotor bars }\end{array}$ \\
\hline 7.5 & 380 & 15.4 & 1440 & 32 \\
\hline
\end{tabular}

The type of test induction motor is Y132M-4, and its main technical parameters are shown in Table 5 . Besides a healthy rotor, the motor is equipped with a fault rotor to simulate the BRB fault. This fault rotor has one broken bar with a drilling hole (diameter: $8 \mathrm{~mm}$ and depth: $10 \mathrm{~mm}$ ), as shown in Figure 5.

There are two experimental conditions, namely, one broken bar under half load $(s=0.02200)$ and one broken bar under full load $(s=0.04133)$. A phase current $i_{\mathrm{a}}$ is used to detect BRB fault, and its waveforms under two conditions are shown in Figure 6. Sampling interval of current signal is $7 \mathrm{~ms}$, and sampling length is 200 . The parameters of IBPSO are the same as that in Section 4.2. In order to compare the results, the traditional MUSIC and DFT algorithm are also used to analyze the measured current signals.

In Figure 7, the results of three algorithms (i.e., MUSIC with search step $\Delta f=0.001 \mathrm{~Hz}$, DFT, and IBPSO-based MUSIC) under full load are compared. As is known, the components of $(1 \pm 2 s) f_{1}$ are the characteristics of BRB fault. Generally, it's relatively easy to find out the fault features under full load, because fault components have highest amplitudes and are furthest away from fundamental component. Thus, three algorithms can all distinguish fault-related components, as shown in Figure 7. However, the distinction effects of MUSIC and IBPSObased MUSIC are more obvious, this is because their calculation principles can avoid the spectrum leakage effectively. Moreover, the algorithms based on MUSIC have better frequency resolution. Although DFT has bigger errors on both fundamental frequency and faultrelated frequencies, it has an absolute advantage on computation time. 


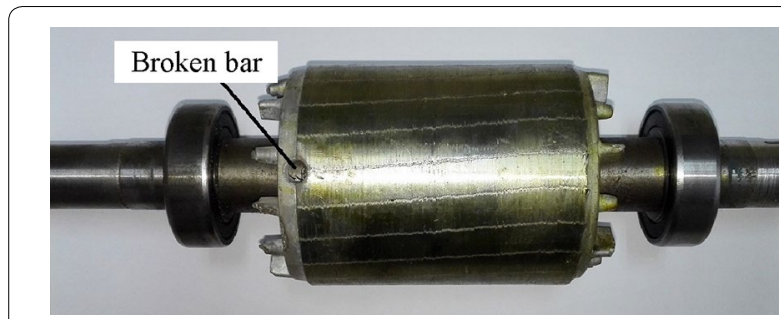

Figure 5 Fault rotor with one broken bar

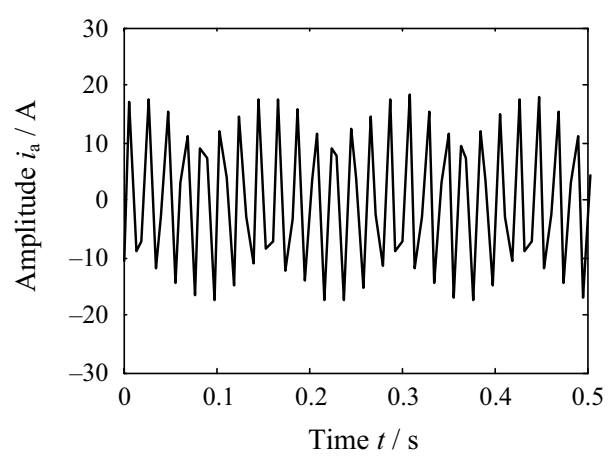

a Half load

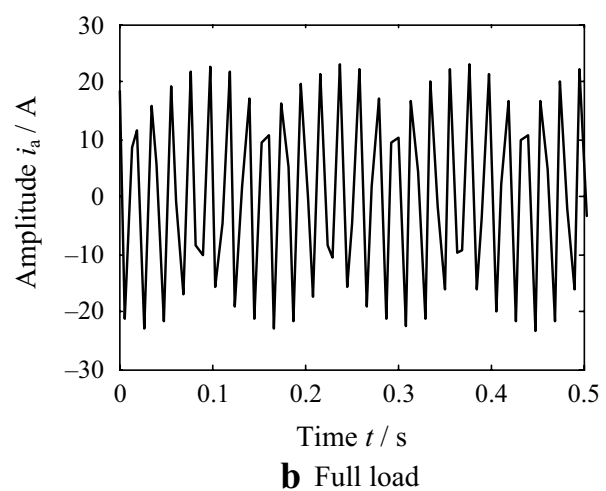

Figure 6 Fault motor currents under different load

Figure 7(a) and (b) show that both two algorithms have a high frequency resolution. However, for traditional MUSIC, spectrum peaks can only appear at the integer multiples of $\Delta f(0.001 \mathrm{~Hz})$ due to usage of fixed step size. As shown in Figure 7(b), three spectrum peaks are only located at $45.861 \mathrm{~Hz}, 50.023 \mathrm{~Hz}$ and $54.224 \mathrm{~Hz}$. Therefore, traditional MUSIC cannot attain a finer frequency resolution. Contrastively, it is obvious that IBPSO-based MUSIC can find higher spectrum peaks no matter for fundamental frequency or for fault feature frequencies (under the same signal subspace and noise subspace). Figure 7 (a) and (b) prove that the frequency resolution and accuracy of IBPSO-based MUSIC are higher than

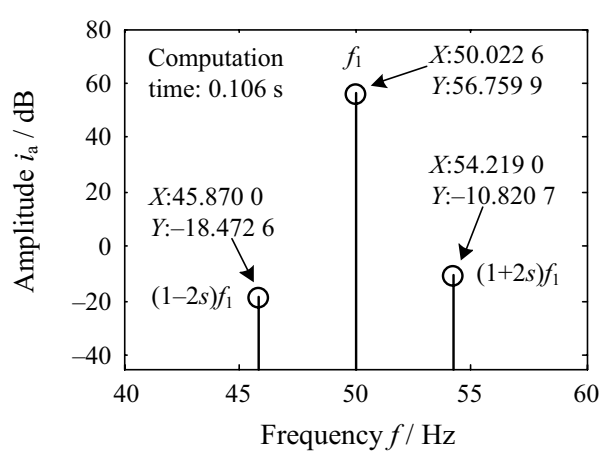

a IBPSO-based MUSIC
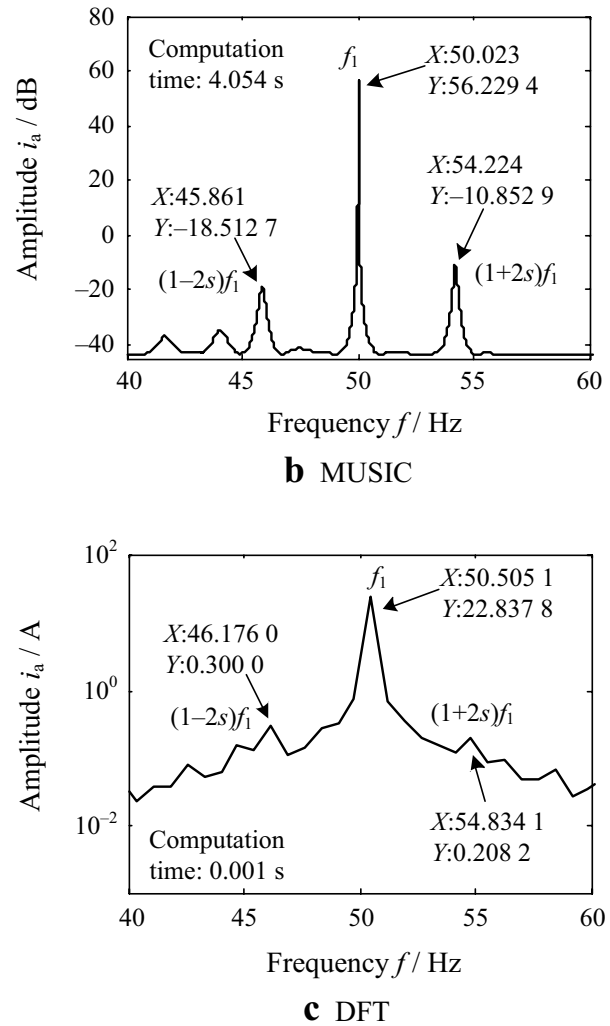

Figure 7 Spectrum analysis results of three algorithms at full-load condition

MUSIC. Meanwhile, IBPSO-based MUSIC also takes a less computation time. In this experiment, its time cost is only $0.106 \mathrm{~s}$ comparing with 4.054 s of MUSIC, which greatly improves the search efficiency. Accordingly, IBPSO-based MUSIC is more suitable and reliable for BRB fault detection.

Figure 8 shows the experimental results under half load. On this occasion, fault components are more weak and closer to fundamental component. So it's more difficult to extract and highlight fault components. Comparing 


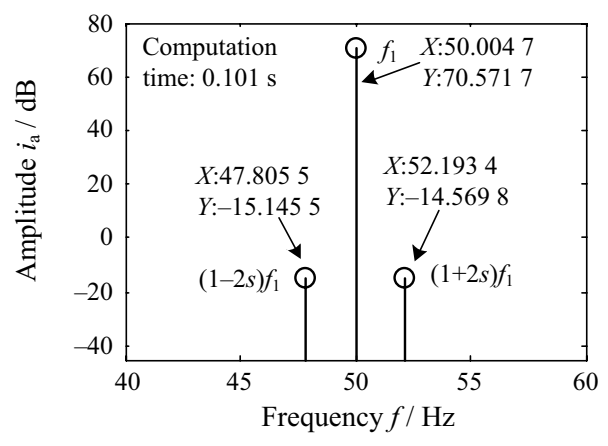

a IBPSO-based MUSIC
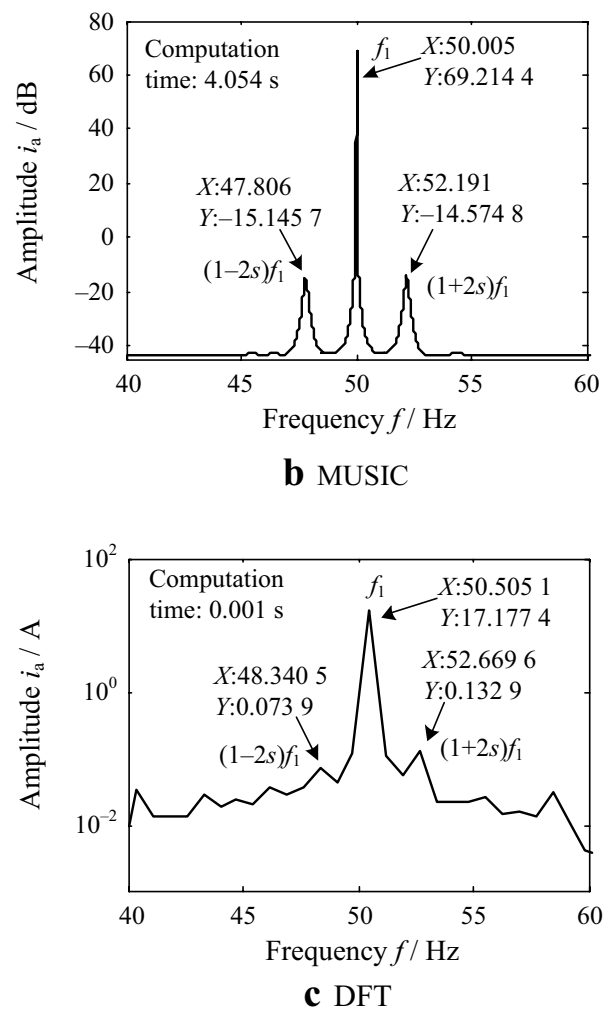

Figure 8 Spectrum analysis results of three algorithms at half-load condition

the results of three algorithms in Figure 8, IBPSO-based MUSIC has the best performance and low computation cost.

\section{Conclusions}

(1) An improved BPSO (IBPSO) is proposed to make it more suitable for multimodal optimization problem. Compared with SCGA and SPSO, the solution quality and convergence speed are improved greatly.
(2) Based on multimodal intelligence search of the IBPSO, a new MUSIC algorithm is proposed for $\mathrm{BRB}$ fault detection. Contrasting with traditional MUSIC, the IBPSO-based MUSIC obtains a prominent improvement on frequency accuracy and search efficiency.

(3) Finally, DFT, MUSIC and IBPSO-based MUSIC are compared in BRB fault detection of an actual induction motor. Experimental results show that the proposed algorithm has the best frequency resolution and accuracy, which make it more reliable to detect the BRB fault.

\section{Authors' Contributions}

P-PW was in charge of the whole trial; P-PW, X-XC and YZ wrote the manuscript; Y-JH and C-XM assisted with sampling and laboratory analyses. All authors read and approved the final manuscript.

\section{Author Details}

${ }^{1}$ School of Electrical and Power Engineering, China University of Mining \& Technology, Xuzhou 221116, China. ${ }^{2}$ School of Information and Control Engineering, China University of Mining \& Technology, Xuzhou 221116, China.

\section{Authors' Information}

Pan-Pan Wang, born in 1982, is currently a lecturer at School of Electrical and Power Engineering, China University of Mining \& Technology, China. He received his PhD degree from China University of Mining \& Technology, China, in 2013. His research interests include fault diagnosis of induction motor, intelligence optimization, signal processing.

Xiao-Xiao Chen, born in 1992, is currently a master candidate at School of Electrical and Power Engineering, China University of Mining \& Technology, China.

Yong Zhang, born in 1979, is currently an associate professor at China University of Mining \& Technology, China. He received his PhD degree from China University of Mining \& Technology, China, in 2009. His main research interests include intelligence optimization and its application, optimal control.

Yong-Jun Hu, born in 1963, is currently an associate professor at China University of Mining \& Technology, China. He received his master degree from China University of Mining \& Technology, China, in 1987. His research interests include signal processing, artificial intelligence, harmonic detection.

Chang-Xin Miao, born in 1976, is currently an associate professor at China University of Mining \& Technology, China. He received his PhD degree from China University of Mining \& Technology, China, in 2012. His research interests include intelligence optimization, smart grid, reactive power compensation.

\section{Competing Interests}

The authors declare that they have no competing interests.

\section{Funding}

Supported by Fundamental Research Funds for the Central Universities (Grant No. 2017XKQY032).

\section{Publisher's Note}

Springer Nature remains neutral with regard to jurisdictional claims in published maps and institutional affiliations.

Received: 27 June 2016 Accepted: 11 September 2018

Published online: 21 September 2018

\section{References}

[1] M Seera, C P Lim, S Nahavandi, et al. Condition monitoring of induction motors: A review and an application of an ensemble of hybrid intelligent models. Expert Systems with Applications, 2014, 41(10): 4891-4903. 
[2] M R Mehrjou, N Mariun, M H Marhaban, et al. Rotor fault condition monitoring techniques for squirrel-cage induction machine - A review. Mechanical Systems and Signal Processing, 2011, 25(8): 2827-2848.

[3] S Bindu, VVThomas. Diagnoses of internal faults of three phase squirrel cage induction motor - A review. Proceedings of the IEEE International Conference on Advances in Energy Conversion Technologies, Manipal, India, January 23-25, 2014: 48-54.

[4] M O Mustafa, G Nikolakopoulos, T Gustafsson. Broken bars fault diagnosis based on uncertainty bounds violation for three-phase induction motors. International Transactions on Electrical Energy Systems, 2013, 25(2): 304-325.

[5] I Culbert, W Rhodes. Using current signature analysis technology to reliably detect cage winding defects in squirrel-cage induction motors. IEEE Transactions on Industrial Applications, 2007, 43(2): 422-428.

[6] V Ghorbanian, J Faiz. A survey on time and frequency characteristics of induction motors with broken rotor bars in line-start and inverter-fed modes. Mechanical Systems and Signal Process, 2015, 54(1): 427-456.

[7] J Pons-Llinares, J A Antonino-Daviu, M Riera-Guasp, et al. Advanced induction motor rotor fault diagnosis via continuous and discrete timefrequency tools. IEEE Transactions on Industrial Electronics, 2015, 62(3): 1791-1802.

[8] P Karvelis, G Georgoulas, I P Tsoumas, et al. A symbolic representation approach for the diagnosis of broken rotor bars in induction motors. IEEE Transactions on Industrial Informatics, 2015, 11(5): 1028-1037.

[9] M Pineda-Sanchez, M Riera-Guasp, J A Antonino-Daviu, et al. Diagnosis of induction motor faults in the fractional Fourier domain. IEEE Transactions on Instrumentation and Measurement, 2010, 59(8): 2065-2075.

[10] G Georgoulas, I P Tsoumas, J A Antonino-Daviu, et al. Automatic pattern identification based on the complex empirical mode decomposition of the startup current for the diagnosis of rotor asymmetries in asynchronous machines. IEEE Transactions on Industrial Electronics, 2014, 61(9): 4937-4946.

[11] D Camarena-Martinez, C A Perez-Ramirez, M Valtierra-Rodriguez, et al. Synchrosqueezing transform-based methodology for broken rotor bars detection in induction motors. Measurement, 2016, 90(5): 519-525.

[12] B Q Xu, L L Sun. Concurrent discrimination of rotor fault and load oscillation in induction motors. Proceedings of the CSEE, 2016, 36(23): 6518-6527. (in Chinese)

[13] P P Wang, L P Shi, Y Zhang, et al. Broken rotor bar fault diagnosis of induction motors using a hybrid bare-bones particle swarm optimization algorithm. Proceedings of the CSEE, 2012, 32(30): 73-81. (in Chinese)

[14] R Puche-Panadero, M Pineda-Sanchez, M Riera-Guasp, et al. Improved resolution of the MCSA method via Hilbert transform, enabling the diagnosis of rotor asymmetries at very low slip. IEEE Transactions on Energy Conversion, 2009, 24(1): 52-59.

[15] CT Kowalski, W Kanior. Effectiveness of the frequency analysis of the stator current in the rotor fault detection of induction motors. Proceedings of the IEEE International Conference on Industrial Technology, Chengdu, China, April 21-24, 2008: 1-5.
[16] A Garcia-Perez, RT Rene, E Cabal-Yepez, et al. The application of highresolution spectral analysis for identifying multiple combined faults in induction motors. IEEE Transactions on Industrial Electronics, 2011, 58(5): 2002-2010

[17] F Fang, S Y Yang, X G Hou. Rotor fault feature extraction of motor faults of induction motor based on a modified MUSIC method. Proceedings of the CSEE, 2007, 27(30): 72-76. (in Chinese)

[18] S H Kia, H Henao, G A Capolino. A high-resolution frequency estimation method for three-phase induction machine fault detection. IEEE Transactions on Industrial Electronics, 2007, 54(4): 2305-2314.

[19] D Camarena-Martinez, R Osornio-Rios, R J Romero-Troncoso, et al. Fused empirical mode decomposition and MUSIC algorithms for detecting multiple combined faults in induction motors. Journal of Applied Research \& Technology, 2015, 13(1): 160-167.

[20] R J Romero-Troncoso, A Garcia-Perez, D Morinigo-Sotelo, et al. Rotor unbalance and broken rotor bar detection in inverter-fed induction motors at start-up and steady-state regimes by high-resolution spectral analysis. Electric Power Systems Research, 2016, 133(1): 142-148.

[21] B Q Xu, L L Sun, H M Li. A detection method for rotor bar fault in induction motors based on high frequency resolution spectrum estimation technique and optimization algorithm. Proceedings of the CSEE, 2013, 33(3): 140-147. (in Chinese)

[22] J Kennedy, R C Eberhart. Particle swarm optimization. Proceedings of the IEEE International Conference on Neural Networks, Piscataway, New Jersey, USA, November 27-December 1, 1995: 1942-1948.

[23] Q Wang, J Chen, J Wang, et al. Structural optimization of composite wind turbine blade under aerodynamic loads. Journal of Mechanical Engineering, 2014, 50(9): 114-121. (in Chinese)

[24] D F Wu, A G Li. Error modeling and compensation approach for threedimensional surface scanning robot. Journal of Mechanical Engineering, 2012, 48(13): 61-67. (in Chinese)

[25] J P Li, M E Balazs, G T Parks, et al. A species conserving genetic algorithm for multimodal function optimization. Evolutionary Computation, 2003, 10(1): 207-234

[26] X D Li. Adaptively choosing neighborhood bests using species in a particle swarm optimizer for multimodal function optimization. Lecture Notes in Computer Science, 2004, 31(2): 105-116.

[27] R O Schmid. Multiple emitter location and signal parameter estimation. IEEE Transactions on Antennas and Propagation, 1986, 34(3): 276-280.

[28] M Clerc, J Kennedy. The particle swarm-explosion, stability, and convergence in a multidimensional complex space. IEEE Transactions on Evolutionary Computation, 2002, 20(1): 1671-1676.

[29] J Kennedy. Bare bones particle swarms. Proceedings of the IEEE International Conference on Swarm Intelligence Symposium, Indiana, USA, April 26, 2003: 80-87.

\section{Submit your manuscript to a SpringerOpen ${ }^{\odot}$ journal and benefit from:}

- Convenient online submission

- Rigorous peer review

- Open access: articles freely available online

- High visibility within the field

Retaining the copyright to your article

Submit your next manuscript at springeropen.com 